\title{
La scrittura dell'esilio: militari ed intellettuali italiani in Catalogna durante il Trienio Liberal $1820-1823$
}

\author{
Agostino Bistarelli \\ Giunta centrale per gli studi storici - Roma \\ agostino.bistarelli@fastwebnet.it
}

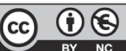

\begin{abstract}
Il lavoro descrive le scritture lasciate dagli esuli italiani che hanno partecipato al Trienio per ricostruirne l'esperienza individuale e il rapporto con la società in cui si calarono. La prospettiva è storica: incrociata con la letteratura storiografica, la loro produzione può dare nuove informazioni sulle relazioni politiche tra Italia e Spagna, sulla vita sociale della penisola nel Trienio, sulle guerre. L'uso di lettere, dichiarazioni testimoniali, memorie e produzione è quindi proposto come problema di analisi della fonti (produttori e destinatari della scrittura, tempi) perché oltre a determinare stili e contenuti, danno informazioni sui processi individuali di costruzione dell'identità che accompagna il percorso risorgimentale.

Parole chiave: Esilio; Trienio liberal; Scritture; Rappresentazioni; Identità.
\end{abstract}

\begin{abstract}
This paper describes texts left by Italian exiles who participated in the Trienio (Triennium) in order to reconstruct their personal experience and relationship with the society in which they were immersed. The perspective is historical: when intertwined with historiographical literature, their production may give new insights into political relations between Italy and Spain, peninsular social life during the Trienio, and wars. The use of letters, witness statements, pleadings and other texts is therefore proposed as a source of analysis (authors and recipients of texts, dates), which, apart from determining style and content, provides information on individual processes of identity construction that took place during the Risorgimento period.
\end{abstract}

Keywords: Exile; Liberal Triennium; Texts; Representations; Identity.

In seguito al fallimento dei moti costituzionali del 1820 nel Regno delle due Sicilie e del 1821 in quello di Sardegna, migliaia di militanti liberali italiani furono costretti all'esilio e una gran parte di essi, attratti dal modello politico e dalla possibilità di continuare la lotta, si diressero verso la Spagna. Questo lavoro affronta le scritture che ci hanno lasciato alcuni di questi esuli per 
ricostruirne l'esperienza individuale e il rapporto con la società in cui si calarono ma in una prospettiva più storica che filologica: incrociata con la letturatura storiografica, la loro produzione può dare nuove informazioni sulle relazioni politiche tra Italia e Spagna, sulla vita sociale della penisola nel Trienio, sulle guerre, quella interna e quella contro i francesi del 1823. L'uso di lettere, dichiarazioni testimoniali, memorie e produzione creativa pone il problema classico della contestualizzazione della fonte con una particolare attenzione, oltre che al produttore, anche al destinatario della scrittura, perché questo ne determina stile e contenuto.

\section{Destinatari e momenti}

E' esemplare il confronto che possiamo fare tra una lettera che Fiorenzo Galli ${ }^{1}$ scrive ai genitori all'inizio dell'esilio e quella che scrive alle autorità piemontesi due anni dopo per chiedere la possibilità di rimpatriare.

Non è possibile esprimere con quale trasporto ci abbiano accolti e quanto sieno ospitali. Niente ci lasciano a desiderare al presente e ci ricolmano della dolce speranza di fare risorgere l'intiera Europa. Felice il Mondo e in particolare il Piemonte se fossero tanti Spagnuoli! La Religione, le proprietà ed il personale sono i più sacri nomi, ed in queste parti inviolabili: il clima, l'opulenza, e mille altre circostanze che qui s'incontrano ci offrono un quadro più perfetto del Paradiso Terrestre. ${ }^{2}$

Una vittima sfuggita alla maledizione del Cielo rifugge al filantropo cuore di V. E., onde impetrare uno scampo. Compromesso nell'ultima rivoluzione di Piemonte, javolò al giusto sdegno del suo oltraggiato Monarca emigrando in Ispagna, ma qual' non fu la sua sorpresa e terrore, appena afferratone il lido, in veè d'essere accolto dalla vezzosa ospitalità, vedersi stendere le braccia dall'orrida peste che nell'atro speco della nera fame precipitosa il condusse! Soltanto il flagello della guerra mancava a porre il colmo alle sue disgrazie e non tardò a coronarle. Rottesi le prime ostilità, per non aggiungere alla taccia di sciagurato quella di vile, si vide nella dura necessita di prendere l'armi. Invitato dal generale in capo dell'armata di Catalogna, venne al suo fianco fin dal principio della spirante campagna ed ottenne da questi in contraccambio delle sue tenui fatiche il grado di Capitano e suo aiutante di campo, impiego che sta tuttavia disimpegnando. Se però le, sue sventure, la sua condotta militare, più volte in pubblici fogli, forse con esagerazione pronunziata, ed il copioso sangue sgorgatogli dalle varie ferite riportate nel campo di battaglia non sono sufficienti per lavare le sue mac-

1. Nato a Carrù nel 1802 da una famiglia giacobina, quando scoppiano i moti era alfiere a Novara. In Spagna è aiutante di Mina e con Luigi Monteggia tra i creatori dell'Europeo. Dopo la fine del Trienio sarà a Londra e in Messico, dove con Claudo Linati edita El Iris. Rientra in Italia nel 1837 e dona a Papa Gregorio XVI la Tabula Philologica, una tavola sinottica con più di 3.000 lingue che secondo lui risalivano ad una unica origine. Muore nel 1844.

2. Lettera di Fiorenzo Galli del 21 aprile 1821 ai genitori, in Archivio dello Stato di Torino, Polizia, Mat. Pol., 1822, II, 8. 
chie e renderlo scevro di colpa agli occhi del suo Sovrano da cui implora la grazia di rimpatriarsi, garantito contro ogni molestia, baccierà la mano che vergasse castigo qualunque sentenza, quando non fosse quella di non poter più mai respirare la dolce aura natia, e consagrare agli adorati suoi concittadini il frutto dei suoi disinganni. ${ }^{3}$

Penso che il riferimento al suo disinganno non sia puramente strumentale e funzionale alla richiesta di perdono, che certo lo influenza, ma non mi sembra la chiave di lettura esclusiva. Analizzando infatti la sua produzione coeva, quella pubblicata su El Europeo e le Mémoires sur la dernière guerre de Catalogne, si può individuare un percorso che dall'entusiasmo iniziale, approda ad riflessione interiore, ad «un continuo ascendere alle idee mistiche e trascendentali secondo la scelta degli estatici» ${ }^{4}$ che segna la produzione inglese (il Prospectus of a Plan of Philosopy e la Tabula Philologica) e quella successiva al rientro in Italia. Penso che il punto di svolta di questo percorso sia il confronto, duro e diretto, con la guerra civile che connota l'esilio spagnolo. Anche in questo caso presento un confronto tra un testo significativo come partenza e uno per l'arrivo.

\section{Prode, Invitto, Immortale, / De' Tiranni e De' Schiavi / Fulmine / Un Proscritto} d'Italia / Questi Carmi / Consacra

\section{CANTO}

Son io d'Apollo nell'augusta reggia,

$\mathrm{O}$ dell'alme Camene in compagnia?

Qual piaggia è questa che si dolce eccheggia

Della più soave e tenera armonia?

Ah! Di Barcino è ' 1 fortunato lido

Di eccelsi vati delizioso nido.

Ma chi motiva lor sublimi accenti?

Ignaro! ... e lo dimando? Il gran Campione

Che dalla strage liberò le genti

Ch'hanno per stemma intrepido leone

Forse a bear non giunge questa sponda

Che immersa stava in doglia profonda?

Alla causa di Spagna non s’innesta

Quella del liberale europeo?

Perciò se un cor riconoscente attesta

Il vate ibero al prode semideo

Egli è dover che al suo benefattore

Inni consacri un italo cantore

3. AST, Consolati nazionali Barcellona, lettera del 25 ottobre del 1823 trasmessa con nota del 18 novembre a La Tour, ministro degli Esteri.

4. PIO CONTI, Inaugurandosi a Carrù la lapide commemorativa in onore del poliglotta prof. Fiorenzo Galli, Carrù: Società operaia, 1902, p. 7. 
Il dolce mormorar di limpid' onda, Del vario pinto angel le soavi note, Il vago aspetto d'una verde fronda

Non son più di mio plettro amena dote:

Solo m'ispira il pallido lamento, La vendetta, il furor, ed il tormento

Terribil squillo! Che in l'orrendo abisso

Li Genii del mal chiamò a consiglio

Per stringer ciò che da natura è fisso.

Ah! non mi sento inumidire il ciglio

Ma roder dalla rabbia allor ch'io miro

Le trame che in quel baratro s'ordiro,

Proposta con terribile ardimento

Viene la tomba d'ogni umano diritto,

E' autorizzato l'empio tradimento

Per far di libertà il più gran conflitto.

Sciolto il congresso alfin, in ogni dove

Volan di lor ferocia a dar le prove.

Ombre amiche, adorate, il grato Eliso

Volgete ad abitar lieti, e contenti;

De' proscritti d'Italia in cor è inciso

Vendicar vostra morte, o cader spenti. ${ }^{5}$

\section{In attestato del più sicuro affetto}

Convinced by ten years of experience, of travels and of trials, by practical and constant study of the great map of the world, that the picture of which I have just given an outline is not exaggerated, and that the political horizon is really gloomy, more gloomy than I am able to depict it, I propose to civilized nations a system of philosophy, the object of which is to attack the very root of the evil, to calm the passions, to dress the wounds, to extinguish the spirit of party, and to promote union, peace and harmony amongst men, by endeavoring to reconcile science with morality, politics with religion, philosophy with divinity, nature with God. It is well known, that without religion the people have no security for the justice of kings, kings for the loyalty of the people, people and kings for the sanctity of priests, nor people, kings and priests, for the favor of the Almighty. The strength of the nations of Europe is already too much impaired by error, unbelief and domestic differences: Woe to these nations! - it is here that the judgment of God awaits them. ${ }^{6}$

5. Fiorenzo Galli, All'inclito Mina, Valencia: Ed. Domingo, 1822. Il riferimento alle ninfe Camene e a versi di Milton o Tasso (Il dolce mormorar di limpid' onda) si spiegano con la sua precocità negli studi classici: «a quattordici anni riusciva a comporre una tragedia nella quale, a giudizio dei contemporanei, apparivano i lampi di un ingegno precoce e peregrino» (Conti, Inaugurandosi a Carrù, cit., p. 6)

6. Fiorenzo Galli, Prospectus of a Plan of Philosopy, London, 1830, p. 21. 
Dunque questi confronti, per tempo e destinatari diversi, ci aiutano a investigare il rapporto tra esilio e scrittura, sul quale però è utile ora fornire elementi di cornice dove inserire il periodo e il luogo che studiamo.

\section{L'addio}

«Ammaina la tua vanità, dice l'esilio, non sei che un granello di sabbia nel deserto. Non ti confrontare con gli altri uomini di penna, ma con l'infinità umana: la quale è umana e triste più o meno quanto quella non umana. $\mathrm{E}$ questo che deve suggerirti le parole». ${ }^{7}$ C'è così un piano soggettivo di questo rapporto, indagato anche dalla psicoanalisi, come nel caso dei Grinberg che, nell'analisi dell'esilio come emigrazione particolare, pongono l'attenzione su alcuni nodi tematici, partendo dal lavoro di $\mathrm{Bion}^{8}$ sul rapporto contenitore —contenuto. L'esiliato visto come idea nuova (il contenuto) e la comunità come gruppo ricevente (il contenitore), attribuiscono al commiato un senso dirimente: "la partenza è il confine che divide lo stato di unione dallo stato di separazione tra chi se ne va e chi rimane, tra la speranza e l'assenza». ${ }^{9}$ Mancare il rito del saluto significa rompere quella protezione, aggiungere all'esperienza già dura una angoscia in più. Sostengono che «molti esiliati possono soffrire della "sindrome del sopravvissuto", sentendosi oppressi dalla colpa provata nei confronti dei morti o dei prigionieri. Questo stato d'animo si trasforma in fertile terreno per lo scetticismo e la delusione». È quindi chiaro l'emergere del viaggio liminare come topos nelle scritture dei nostri esuli. "Addio dunque, terra diletta, amatissimo albergo d'ogni a me più cara cosa, ove le prime aure ore di vita respirai, ove sperava chiudere i giorni in pace. Addio, patria adorata, scopo dolcissimo a tanti fervidi voti, a tante care speranze». ${ }^{10}$ Secondo Giovanni Albertocchi questo appassionato passo dell'esule piemontese «anticipa el que uns quants anys més tard, en la famosa novel.la d'Alessandro Manzoni, I promessi sposi, Lucia recitarà en abandonar el paìs natal», addio che risuonerà anche, com'è noto, nel poema La pàtria di Carles Aribau. ${ }^{11} \mathrm{Mi}$ interessa sottolineare questo rimando a spirale perché è analogo a quello che si ritrova continuamente nelle vicende degli esuli italiani: per tutti valga il circuito che parte da Ugo Foscolo che esule a Londra studia Dante e che passa per tutti gli esuli successivi nella città e che studiano, pubblicano, commentano l'opera dei due e che costituiscono l'ossatura di quello che è stata definita una

7. Josif Brodskij, Profilo di Clio, Milano: Adelphi, 2003, p. 45.

8. E in particolare Wilfred R. BION, Attention and interpretation, London: Tavistock Publications, 1970.

9. Leon Grinberg - Rebeca Grinberg, Psicoanálisis de la migración y del exilio, Madrid: Alianza editorial, 1984, p. 161, anche per la citazione successiva.

10. Carlo Beolchi, Reminiscenze dell'esilio, Torino: Biancardi, 1853 [ma London, 1830], p. 23.

11. Giovanni Albertocchi, «Exiliats italians a Catalunya a començament del segle XIX», Revista de Catalunya, n. 215, 2006, p. 20. 
istituzione italiana. ${ }^{12}$ Torniamo però alla partenza per l'esilio e a come è stata raccontata in esuli diretti in Spagna, fornendo alcune tipologie narrative.

Tramontava il sole del 17 aprile 1821 quando dicemmo l'ultimo addio agli amici ed alla patria. Salpammo per Barcellona. Io col cuore rotto dal dolore mi posi a giacere sul ponte del vascello, e mi sorgevano l'una appresso l'altra tante immagini che mi funestavano l'animo, tra le quali l'abbandono della famiglia. Ma una fiera burrasca le dileguò. ${ }^{13}$

Le navi cariche di tanti sventurati salparono; quelle più veloci della nostra sorpassandoci pareva volessero fermarsi per stringerci la mano. La nostra costernazione era al colmo. A noi nullamente assuefatti a coteste vicissitudini e novizi ai viaggi sul mare era troppo doloroso allontanarsi dalla Patria. Quasi tutti pativano il mal di mare. Alcuni colle braccia conserte stavan meditando e guardando l'ognor crescente spazio che ci allontanava dal Lido; molti per calmare il loro cordoglio si misero a cantare: Addio Padre Addio Madre Addio tutti non vi vedrem mai più; se la mia morosa è una bella tosa la pregherà per me; Profezia avveratasi per molti! Tutti si attristavano di separarsi dalla patria per andare in terra straniera, ove ci si poteva chieder conto della nostra condotta, e rimproverarci di non aver saputo morire in difesa del patrio suolo. ${ }^{14}$

Si fece vela verso la Spagna; e sarebbe troppo lungo e doloroso il voler qui ricordare la posizione critica in cui il bastimento e noi ci trovammo durante i primi cinque giorni di navigazione verso Barcellona ... Il mare essendosi intanto calmato, ci fu dato di arrivare, nel termine di quattro giorni, al nord della costa del porto di Rosas, d'onde facendo vela verso il mezzodì e costeggiando il lido spagnuolo a poca distanza da esso, ci dirigemmo al porto di Barcellona, dove approdammo verso le due pomeridiane del 19 aprile..$^{15}$

Io aveva scelto per mio rifugio le montagne della Svizzera aspettando che passasse il temporale. Ma avendomi il ministro B. , con cui da lungo tempo sono stretto in amicizia, invitato a recarmi seco lui in Ispagna non esitai punto a cambiare una dubbia ospitalità con un asilo sicuro. Eccomi adunque quasi per incantesimo trasportato qui da un bel Landau a tira sei. ${ }^{16}$

Negli esuli la necessità di accettare una sconfitta, nella ricostruzione della vita quotidiana, non rende semplice il processo di integrazione, anche perché questa rottura della sacralità dell'esilio può generare la paura della perdita di identità. L'integrazione può essere sentita come un tradimento e questo può trasformarsi in un rifiuto della comunità ospitante. Il considerarsi di passaggio di alcuni esuli spiega

12. Carlo Cattaneo, «Ugo Foscolo e l'Italia», Il Politecnico, 1860.

13. Giuseppe AvezZANA, I miei ricordi, Napoli: Fibreno, 1881, p. 29.

14. Giuseppe Sorisio, Memorie di Sorisio Giuseppe emigrato dal 1821, manoscritto conservato nel Museo Nazionale del Risorgimento di Torino, Archivio Museo, p. 19-20.

15. Pietro Garda, La rivoluzione del 1821, Ivrea: Curbis, 1879, p. 42.

16. Giuseppe Pecchio, Sei mesi in Ispagna nel 1821; Lettere di Giuseppe Pecchio a Ledi G.O., Madrid, 1821 nella edizione contenuta in ID, Scritti politici, a cura di PAOlo BERNARDELl, Roma: ISRI, 1978, p. 3-50, p. 6. Il riferimento è a Eusebio Bardaxi y Azara già ambasciatore a Torino e poi ministro degli esteri spagnolo. La lettera è datata Irún, 5 maggio 1821. 
la mancanza di interesse a raggiungere il precedente livello sociale o professionale; allo stesso tempo la degradazione sociale di molti esiliati aumenta la loro insicurezza e il senso di persecuzione. Il bisogno di svolgere, per sopravvivere, i lavori più diversi che non hanno nulla in comune con il lavoro svolto in patria - in una situazione di eccessiva dipendenza dagli altri che contrasta con la precedente indipendenza - rischia di suscitare nell'esiliato sensazioni di depersonalizzazione, dal momento che gli è difficile assumere un'identità diversa da quella di «esiliato». ${ }^{17}$

\section{Esilio e scrittura}

Queste osservazioni aiutano a capire l'importanza cha ha assunto l'insegnamento dell'italiano e la produzione pubblicistica per molti degli esuli dei moti risorgimentali, strumenti per la sopravvivenza materiale, ma anche per rimanere ancorati al proprio mondo. Del rapporto tra esilio e lingua danno visioni complementari poeti (l'esilio «è, prima di tutto, un evento linguistico: uno scrittore esule è scagliato, o si ritira, dentro la sua madrelingua. Quella che era, per così dire, la sua spada, diventa il suo scudo, la sua capsula»), ${ }^{18}$ e storici: «in alcuni tipi di migrazione la scrittura è insieme strumento per gestire il cambiamento culturale e arma per lasciare una traccia del proprio vissuto». ${ }^{19}$

L'intreccio tra produzione politica degli esuli e dinamiche locali è così ricordato:

La literatura fulletonesca feta pels italians exiliats a Espanya durant el Trienni Constitucional mereix un paràgraf. Foren molts els pamflets, fulletons $\mathrm{i}$, fins $\mathrm{i}$ tot, algun opuscle polític que es publicaren a Barcelona, Madrid o València. A la ciutat comtal la família Roca es convertí en la impressora de totes les obres elaborades pels italians. Els diaris de la ciutat també permeteren que en les seves pàgines apareguessin articles signats pels italians residents a Catalunya. Tot aquest material i el publicat en d'altres ciutats espanyoles ens permetrà esbrinar quin discurs polític forgen i com es relaciona amb la cultura política imperant a Barcelona. ${ }^{20}$

Rilevante è quindi il ruolo italiano in questa radicalizzazione dell'alfabetizzazione politica che poi, come rileva Alberto Gil Novales, precipita — una volta che le classi dirigenti percepiscono il pericolo- in una controrivoluzione di ambito continentale. ${ }^{21} \mathrm{E}^{\prime}$ interessante ricordare quello che uno degli autori a

17. GRINBERG, cit., p. 163.

18. Josif Brodskij, Dall'esilio, Milano: Adelphi, 2001, p. 32.

19. Camillo Brezzi-AnNa Iuso, Introduzione a «Esuli pensieri», Storia e problemi contemporanei, n. 38, a. XVIII, 2005, p. 8.

20. JORDI ROCA VERNET, La Barcelona revolucionària : cultura constitucional i la sociabilitat política del Trienni Constitucional : 1820-1823, Tesi UAB, 2005.

21. Il tema è presente in più parti di AlBerTo GIL NovalEs, Las sociedades patrioticas, 18201823 : las libertades de expresion y de reunion en el origen de los partidos politicos, Madrid: Tecnos, 1975, 2 voll. 
cui si riferisce Jordi Roca, Emilio Bottone, scrive al fratello Alessandro della comunità degli esuli liberali a Barcellona,

dove intraprenderemo la pubblicazione di un giornale italiano intitolato: il Costituzionale Italiano. Un simile periodico che si stampava a Londra contribuì non poco al ristoramento della Costituzione in Spagna: non mancare dunque di mandarmi quante notizie ti possono pervenire e che facciano pel caso nostro; puoi anche talora spedirmi i giornali Inglesi che ti paiono interessanti; poiché sebbene non sappia io scrivere, ne parlare questa lingua tuttavia la capisco. Tu potresti ancora promuovere costì una simile impresa. Scrivimi se si crede che gli Inglesi siano per dar favore alle cose d'Italia. ${ }^{22}$

Rimandando per l'analisi in particolare dei contributi politici di vari esuli, tra i quali spicca Bartolomeo Fiorilli, ai lavori di Jordi Roca, ${ }^{23}$ riprendo l'esempio di cosa può indicarci l'incrocio di queste scritture con la letteratura storiografica e la cronologia degli avvenimenti. Prima però mi sembra interessante segnalare una riflessione a proposito della lingua, o meglio delle lingue, incontrate in questo esilio:

La lingua de' Barcellonesi è la catalana, sebbene i signori adoprin anche la castigliana, massime conversando cogli stranieri. La differenza tra queste due lingue è grande, ed un Catalano del volgo non capirebbe il castigliano più presto che l'italiano o il francese. La lingua catalana in confronto alla castigliana si può dir dialetto, ma considerata in se stessa è vera lingua, ricca, robusta, e non senza certa leggiadria che le viene dai suoi diminutivi di cui abbonda. Essa ha grammatica, dizionario, scrittori di prosa e di verso, ed ha servito agli atti pubblici. Un Italiano, che la sente per la prima volta, è piacevolmente sorpreso di poterla capire quasi interamente, trovandovi molte voci italiche, o de' dialetti d' Italia. Colui poi che ha letto gli scritti di que' signori della scienza gaja, i quali coll' armonia de' loro canti ivan dirozzando i barbari costumi dell' undecimo e duodecimo secolo, voglio dire de' Trovatori, vi scorgerà con maraviglioso diletto una medesima lingua, od a quella somigliantissima. ${ }^{24}$

22. La lettera è datata 30 ottobre ed ha questo inizio: «Caro fratello e più che fratello amico, Dacché tu mi lasciasti non ebbi che due sole lettere tue, una da S. Sebastiano e l'altra da Bilbao alle quali non ho lasciato di rispondere; sebbene io credessi che le lettere mie non fossero per giungervi in tempo. Di tutte quelle somme che tu graziosamente mi cedesti io non riscossi un soldo solo; è però vero ch'io non volli molestare nessuno». Conservata nel Fondo Buon Governo segreto dell'Archivio di Stato di Firenze e citata quasi integralmente da ERNESTO BENEDETTO, "Cospiratori piemontesi in Toscana ed un fallito attentato alla vita di Carlo Alberto in Pisa nel 1822», in, Rassegna storica del Risorgimento, 1936, p. 1197 1218, p. 1203-1206.

23. Oltre la tesi citata vedi De la democràcia a Napoleó. Les grans aportacions dels exiliats italians al discurs politic liberal del Trienni Constitucional (1820-1823), in Congrés "Ocupació i resistencia a la Guerra del Francès (1808-1814)», Barcelona (5-8 octubre 2006), vol. 2, p. 1063-1087.

24. BEOLCHI, Reminiscenze, cit., p. 113. 


\section{Scrittura come fonte tra altre fonti}

Con il nuovo governo San Miguel, anche sull'onda del pericolo scampato a Madrid dopo la rivolta della Guardia Real (7 luglio), si passa a contromisure decise come la dichiarazione dello stato di guerra e l'incarico ad Espoz y Mina a comandante dell'esercito di stanza in Catalogna. A questo proposito, oltre ai versi di Galli già citati, posso indicare le parole di Pecchio che quando Mina aveva iniziato le operazioni in Catalogna ricorda: «the factious fly at his approach as birds shun the hawk». ${ }^{25}$ Anche Sorisio testimonia delle speranze suscitate nel fronte costituzionale: «I liberali inebriavansi di gioia» per la nomina di Espos Y Mina a Capitano generale della Catalogna». ${ }^{26}$ Abbiamo infine un altro ricordo, quello di Giovanni Allegra che scrive:

La Spagna è tutta commossa; molte dissensioni, bande di insorgenti e mal affetti al sistema attuale di governo compaiono in pressoché tutti i punti della Penisola; ma poche guardie nazionali, poca truppa disperdono il maggior numero degli avversari, e si spera con ragione la calma all'installazione delle Cortes. Intanto poco mancò che ai 24 non corresse qui in Barcellona il sangue cittadino per una quistione nata tra il Capo Politico e il Colonnello della Guardia Nazionale; ma per ventura si sedò il tumulto, e tornò la dolce calma senza che avvenisse disgrazia. State sano e salutate la carissima madre. ${ }^{27}$

Ma come era vista in generale l'accoglienza? Ci aiuta ancora Giuseppe Pecchio, con un brano rimasto poi famoso nella storiografia sul periodo.

In Barcellona, Tarragona e Valenza si trovano da ben cinquecento rifugiati piemontesi. Le Corti hanno decretato che si diano loro dei sussidi mensili in proporzione dei gradi e delle cariche che occupavano sotto il sistema costituzionale. La Spagna si è condotta cogl'italiani come colui che lascia assassinare il suo amico senz'accorrere in suo aiuto, ma poi lo ricovera in casa per medicargli le ferite. ${ }^{28}$

Di quei primi giorni abbiamo anche notizie da Giuseppe Bruno, ventenne di Alessandria che poi però torna subito in Italia dove racconta che nella città catalana riceveva una pezzetta al giorno, che era alloggiato in un quartiere con altri 35 tra sottufficiali e soldati, mentre gli ufficiali potevano stare in città e che avevano ricevuto la paga per quindici giorni in relazione al grado. $\mathrm{Nel}$ rapporto che raccoglieva la sua testimonianza ci sono indicazioni assai interessanti: «in Barcellona sono stati ricevuti piuttosto bene che male, ha recato però

25. Giuseppe Pecchio, Journal of Military and Political Events in Spain during the last twelve months, London: Whittaker, 1824 edizione contenuta in ID., Scritti politici, cit., p. 89-151, p. 112.

26. Sorisio, Memorie, cit., p. 46.

27. Gian Luigi Colli, L'avvocato Giovanni Allegra da Costigliole di Saluzzo: note storiche e biografiche: dal 1821 al 1861, Torino: Bona, 1886, p. 55.

28. PECCHIO, Sei mesi in Ispagna nel 1821, cit., p. 25. 
molta sorpresa il loro arrivo ai Barcellonesi, i quali dicevano che non bisognava fuggire, ma combattere sino all'ultima goccia di sangue. Questo carico si fa maggiore ai Napoletani». ${ }^{29}$ Il sentimento doveva essere abbastanza esteso se il 30 aprile il «Redactor General de España» pubblica, con «cierta conmiseración simpática, y no sólo ironía», ${ }^{30}$ queste rime:
Pulcinella malcontento
Disertor del Reggimento
Scrive a mamma in Benevento
Della Patria il tristo evento:
Movimento, Parlamento,
Giuramento, giuramento, gran fermento, poco argento, armamento, e nel cimento, tra spavento e tradimento, me ne pento, me ne pento, siam fuggiti come il vento. Mamma cara, mamma bella Prega Iddio per Pulcinella

È interessante notare questa immediata ripresa dalla canzonetta variata, secondo Croce, da Giovanni Giraud su un tema classico precedente - quello della stanchezza della guerra- che accomunava tutte le maschere, ${ }^{31}$ ma che dopo il moto del 1820 assumeva un senso particolare, quasi a rappresentarne il fallimento. ${ }^{32}$ Non a caso a Napoli comparve anche la risposta della mamma all'esortazione finale di Pulcinella, che nel sarcasmo velenosissimo può essere lo specchio di sentimenti più generali:
Donna Chiara immantinente
Rispondendo amaramente
Al figuolo delinquente
$F e$ ' la replica seguente:
Ch'aggio inteso, figlio infame
Tu sia acciso dalla fame, Che ti possa magnar l'orco!
Sei fuggito come un porco,
Hai tradito il parlamento,
Tu con tutto il reggimento.
Ah! managgio, che minchioni

29. Rapporto del 16 giugno 1821 citato da MARIO DOSSENA, «Lettere di esuli piemontesi del 1821», Rivista di storia arte archeologia per le province di Alessandria e Asti, 1981, p. 77-91, p. 88-89.

30. Gil Novales, Las sociedades patrioticas, cit. p. 621.

31. Anton Giulio Bragaglia, Pulcinella, Firenze, Sansoni, 1982 (ma 1953), nel capitolo XXIII dal significativo titolo Il cittadino Pulcinella, ricorda ad esempio lo stesso motivo per Arlecchino.

32. Benedetto Croce, Canti politici del popolo napoletano, Napoli : Priore, 1892, p. LXVII. 
Che vuon far costituzioni

Quando ad altro non son buoni

Che a mangiar dei maccheroni

E cacarli nei calzoni.

Figlio unico, figlio caro,

Dio t'assista, e S. Gennaro;

Carbon spento è fuoco ito.

Pelle gambe non c'è male,

Il ginocchio guarirà.

S'è l'Italia uno stivale

E tal sempre resterá ${ }^{33}$

Analogo incrocio può esser fatto per le vicende che riguardano lo scontro con la reazione interna o quello con i francesi nella guerra del 1823 , vedendo come le memorie degli esuli abbiano contribuito a costruire un pantheon dell'esperienza per poter rivendicare il proprio contributo al percorso di costruzione della nazione. A titolo di esempio possiamo descrivere la figura di Giuseppe Pacchiarotti, già ufficiale nel reggimento di Alessandria, nato nel fatidico 1789 a Castelnuovo Scrivia. Ferito nel combattimento di Lladó venne poi portato all'ospedale di Perpignan, dove rifiutò l'amputazione di una gamba e morì dopo 12 giorni di agonia.

Grande della persona, di nobile e grato aspetto [...] Coloro che l'han veduto combattere, non dimenticheranno mai la serena sua fronte in mezzo ai pericoli, e la sua ferocia negli assalti. Vinceva col valore i nemici, colla piacevolezza e cortesia i compagni. I Francesi che militavano al suo fianco solevan chiamarlo: Le brave Pièmontais. Quelli che lo combattevano e gli erano nemici avevan per lui la più alta stima e considerazione. Amò la patria e la libertà sopra ogni cosa. Fu tacciato di ambizione: era smisurato desiderio di gloria, a conseguir la quale non è cosa che non avrebbe osato. Il suo nome vive e vivrà lungo tempo venerato in Catalogna e Spagna. Se la fortuna gli avesse aperto più vasto campo che non il comando di pochi esuli, avrebbe operato grandi cose, e lasciato un nome fra gl'illustri capitani d'Italia. ${ }^{34}$

A lui è dedicata anche una vasta produzione agiografica e il riferimento all'ambizione è forse dovuto all'episodio del 7 luglio 1821 quando era a capo della commissione per determinare il grado di soccorso,

mentre attendeva a sì delicato incarico, due suoi concittadini, già ufficiali dell'esercito piemontese, credutisi mal classificati, e per gelosia, dal Pac-

33. Rimproveri fatti dalla mamma, che segue la lettera di Pulcinella, nella versione contenuta in La coda. Almanacco nato fatto pel 1865 che contiene un po' di tutto e molte altre cose serie e da ridere, Bologna: Tip. S.M. Maggiore, 1864.

34. Aneddoti militari, p. 96. 
chiarotti, recavansi armati al suo domicilio, e davano origine ad una rissa in cui l'illustre patriotta, difendendosi, ne stendeva morto a terra uno dopo aver egli riportate parecchie ferite. L'opinione pubblica assolveva il Pacchiarotti, il quale anzi, allo scoppiare in Catalogna della ribellione reazionaria contro le Cortes e la Costituzione, venne dal celebre generale spagnuolo Mina nominato capo della Legione Italiana, creata a combattere gli assolutisti. ${ }^{35}$

La figura di Pacchiarotti entra così nel mito risorgimentale tanto da essere citato anche da grandi protagonisti: se Garibaldi lo cita nel suo romanzo storico Cantoni il volontario, ${ }^{36}$ Mazzini gli dedicherà addirittura alcuni suoi versi giovanili. ${ }^{37}$

\author{
Ai martiri della libertà \\ O Pacchiarotti! Tu peristi e voi \\ Prodi seco periste ... Itali veri \\ Cosi morian! Posate in pace o forti: \\ Voi più non siete, ma nel mondo suona \\ Il nome vostro eterno. \\ O voi beati! \\ Infelice chi resta! Ed io rimango \\ A lagrimar su voi sommessamente! \\ Ch'altro poss'io? Ma d'infecondo pianto \\ Non v'appaga il tributo; il sangue vostro \\ Vendetta chiama e l'otterrà [...]
}

35. Gian Luigi Colli, L'avvocato Giovanni Allegra, cit, p. 59: «difese l'Allegra l'inquisito davanti al Tribunale di Guerra della Catalogna, e così splendida fu la sua arringa, che l'Ufficiale venne pienamente assolto, e il giovane avvocato ebbe lode e fama di valente oratore». Ma Allegra non riesce a fare l'avvocato per problemi di lingua e conoscenza delle leggi locali. Scriverà al padre che lo spingeva a cercarsi un reddito: «Non sarei sincero se non confessassi il mio stato di indigenza; ma non è colpa mia, e si può assicurare con fondamento che l'antico proverbio "la povertà non è vizio" quadra perfettamente al caso mio; voi vi ricorderete dell'ultima vostra, e vi ricorderete dell'avviso datomi — di pensare a me stesso- io non l'ho seguito perché mi trovava in Barcellona, e voi avete rettamente consigliato, perché vi trovavate in Piemonte; sicchè vi prego per quell'amore che sempre aveste per me a non volermi abbandonare, il che spero non farete, giacchè non mi abbandonereste in circostanze per me più critiche. Voi mi incolperete per non aver io lasciato la Spagna prima d'ora; vi risponderò che la Spagna promise dei soccorsi agli emigrati: e perché attesi il compimento delle promesse di una Nazione, rimasi costì fino adesso; in questo poi la colpa è mia e mi rassegno sin d'ora alla penitenza lunga de' miei trascorsi» (1 aprile 1823 , p. 60).

36. Pacchiarotti è tra coloro che appartenevano «alla schiera de' prodi che traditi da uno spergiuro emigrarono nella Spagna» e "che vi fecero bello il nome Italiano, pugnando per gli stessi diritti che avean loro fruttato l'esilio» (capitolo XXX dedicato ad Avezzana, Milano: Politti, 1870, p. 180).

37. Poesie giovanili; con prefazione e note di Arturo Salucci: Milano, Lavinia, 1926. 
Anche Linati si esercitò nella poesia, consacrando "quattro rime» 38 a Pacchiarotti:

Gia delle pugne il tuono orrendo tace

Sul prode estinto per la patria, e il frale

dello schiavo de' regi il Corvo edace

Già batte l'Ale.

[...]

Piangi Italia i tuoi prodi, i figli tuoi

Che l'avara coperse estrania terra

Piangi i tuoi difensor, piangi gli Eroi

Caduti in Guerra

E grata i nomi lor narra alla fama

Ene' sospiri al suon l'età crescente

L'ufficio compi, e paga sia la brama

Dell'uom morente.

Torniamo alle memorie degli esuli sul primo periodo, prendendo come base quelle di Garda. ${ }^{39}$

\section{La peste}

«A quest'epoca tutte le piccole città della Catalogna lungo la strada da noi percorsa e lungo le rive del mare, davano rifugio ad un buon numero di emigrati nostri compatrioti». ${ }^{40}$ Causa di quella disseminazione è il sovrapporsi della necessità politica di non concentrare nello stesso punto gli esuli italiani (sulla quale poi torneremo) e quella di sfuggire all'epidemia di febbre gialla. Questa apparve nella capitale catalana nei primi giorni di agosto e per circa un mese non ci fu la piena coscienza della sua pericolosità, ${ }^{41}$ fin quando tutti

38. Lettera di Linati a Panizzi, da Avignone 6 settembre 1824, in Lettere a Panizzi, British Library, al n. 16. La lettera ci fornisce anche indicazioni preziose sullo stato d'animo dell'esule: «Alcuni riguardi di famiglia mi hanno fatto rimanere in Francia, per non avere l'aria di dare un calcio alla vecchia, quantunque persuaso e persuasissimo che tutte le circospizioni non mi servano a niente in Ispagna. Colà si vuol confiscare, ed è ciò che hanno conseguito con un processo che mi fanno che finirà col medesimo risultato di quello di Parma. In questo stato di cose aveva determinato di passare nel Belgio a provare se colà potessi guadagnarmi il pane ma la mancanza di denari mi ha impedito di farlo, e sto vegetando in questa infamissima terra Papale».

39. Pier Alessandro [Pietro] Garda, Memorie 1815-1860, Ivrea: Ferraro, 1993 e Id., La rivoluzione del 1821, Ivrea: Curbis, 1879.

40. GARDA, La rivoluzione del 1821, cit., p. 47.

41. Nelle memorie degli esuli si fa riferimento alla vana opera di denuncia del medico Simonda, che abitando alla Barceloneta ebbe l'occasione di visitare un marinaio proveniente dall'Avana «e subito riconobbe i segni di quel male che vien detto comunemente la febbre gialla; e senza frapporre indugio ne diede avviso al capo politico di Barcellona» (CARLO BEOLCHI, Reminiscenze dall'esilio, Torino: Tipografia Nazionale, 1852, p. 71). 
i quartieri della città non vennero contagiati ad inizio settembre. «A diecisiete de ese mes la Junta Superior de Sanidad de Cataluña pubblicaba un bando por el cual imponía un cordón sanitario a Barcelona, medida, ésta, que no consiguió frenar su avance hacia otros puntos de Cataluña.. ${ }^{42}$

Scriveva al fratello un esule da Villanueva nel dicembre 1821 di avere buona salute,

ma però sono annoiato di stare in questo piccolo paese (...) Barcellona incomincia a respirare; alli 25 novembre si cantò il Te Deum ed alli 20 dello scorso dicembre si permise di entrare in città: si fanno ascendere a quindicimila le vittime di questo terribile contagio e fra esse settecento guardie nazionali, due alcaldi, quindici medici e diciannove de' nostri emigrati. Tra questi ultimi però, tranne Rattazzi e Appiani, non ve n’è nessuno di tua conoscenza. ${ }^{43}$

Il ricordo dell'epidemia è un elemento costante nelle narrazioni e rimanda alle osservazioni di Romero Marín. ${ }^{44}$ Scrive Pietro Garda: «osservai che Barcellona, afflitta dall'epidemia, aveva perduto tutta la sua vita di attività, e nell'aspetto di tutti i cittadini che incontrai per istrada si leggeva la tristezza e la paura». ${ }^{45}$ La descrizione più interessante del periodo, vista con gli occhi di un italiano, è quella che ci restituisce la lettera di Emilio Bottone al fratello Alessandro:

Si conteranno ora a stento trenta emigrati in Barcellona e poco più di trentacinquemila abitanti di cento ottanta ch'egli erano, sia perché molti si sono prima d'ora in varie parti dispersi, sia perché il morbo crudele che va di giorno in giorno imperversando distrusse non pochi di quelli che rimanevano. ${ }^{46}$

Dopo avergli dato notizie sugli esuli (una trentina rimasti, venticinque morti) e sulle persone di loro conoscenza (tra cui lo stampatore Doria) passa a commentare la triste situazione:

Ma per ritornare al proposito nostro ti dirò che la descrizione lasciataci dal Boccaccio della peste di Firenze è debole immagine delle stragi che fa ogni giorno la morte nel recinto di queste mura, tuttavia siccome io non voglio mettermi in confronto con sì grande autore e che temo il paragone, così mi

42. JoAQUim Bonastra Tolós, Ciencia, sociedad y planificación territorial en la intitución del lazareto, tesis doctoral Universitat de Barcelona 2006, nella versione digitale all'indirizzo www.tdx.cbuc.es, p. 190-191.

43. Carlo Braggio, La rivoluzione piemontese del 1821.

44. Juan Romero Marín, "La dramaturgia social. Dios y fuego en la Barcelona del siglo XIX», Hispania Nova, n.1, 1998-2000, nella versione digitale all'indirizzo www.hispanianova. rediris.es. L'articolo ricorda le relazioni tra l'impatto dell'epidemia e le differenziazioni sociali e anche il rogo in effigie di un medico come rito purificatorio avvenuto nella Rambla «dramatización catártica liberadora como la de Don Carnal».

45. GARDA, La rivoluzione del 1821, cit., p. 48.

46. Lettera di Emilio ad Alessandro Bottone, cit. 
asterrò dal parlarne, e solo accennerò così di passo che pare che la febbre gialla, i medici, gli speziali, i preti ed i frati abbiano fatta qui una santa alleanza intesa ai danni del genero umano. Di fatti, come prima uno cade ammalato, i parenti suoi si danno ogni premura di convocare nello stesso tempo el Doctor Matatodos, fra Oliosanto e D. Rafael Encaxa con i chiodi. Questi non tengono l'infermo molto tempo in disagio ed incomincia il Dottore a scrivergli un medicamento così efficace che subito dopo il primo cucchiaio incomincia l'infermo a perdere immediatamente la parola e poco stante anche i sensi, subentra allora Fray Oliosanto a borbottare colla bocca piena di aromati alcune parolaccie in lingua peregrina e quindi lo rimette nelle mani di Don Rafael Encaxa il quale gli presta brevemente i suoi offici e si dilegua. È severamente proibito di dare più di un medicamento ad un infermo e vi è una tariffa per tutti gli ammalati: una pezzetta (peseta) al medico; una allo speziale, otto per la cassa e un duro e mezzo per Don Rafael il quale è tenuto a provvedere i chiodi ed a portarsi via il cadavere che diventa sua proprietà. Dopo questo vedresti i suoi parenti a spalancare tutte le finestre della casa, ardere incenso, mirra, aceto e varie misture antipestilenziali, lavare accuratamente con aceto tutti gli angoli della casa e, brevemente, a gettare dalla finestra tutti gli oggetti di vestiario che appartenevano al defunto rinunciando a chi la vuole a questa parte della sua eredità! Con molto impegno sono finalmente riuscito a procurarmi una ricetta di questo meraviglioso rimedio, il quale, se dobbiamo giudicare dagli effetti, deve essere un veleno potentissimo che potrebbe per avventura venire in concio quando che sia per l'amato nostro Carlo Felice. S'è veduto qualche raro esempio di persone che sono guarite dalla febbre gialla, ma di tutti quelli che ebbero ricorso a questo rimedio non un solo è scampato; vedi efficacia!! Insomma, questa misera città pare essere trasformata in lagrimarum valle: le strade, le piazze e le passeggiate deserte, i templi abbandonati, i caffe, i teatri, le botteghe chiuse: le campane, i tamburi muti, e se pur vedi di quando in quando qualche viso umano ti si appresenta con aspetto di ombra o di fantasma che ti fa arricciare il pelo per ogni dove. ${ }^{47}$

La figura del dottor Matatodos sembra ispirarsi ai giudizi critici che il dottor Carlo Simonda esprime sui metodi locali. In una relazione fatta per il Jefe político (e che il console piemontese trasmette in Italia), l'esule medico avvertiva che le sole cure usate dal dottor Campany nel lazzaretto erano somministrazioni di oppio, vino, china ed etere: "Il dottore Rusch, che ottenne cosi felici cure in America, sarebbe ben lontano dall'additare un consimile metodo ed il dotto, l'immortale professore Tommasini lo condannerebbe altamente». 48

47. Ibid.

48. AST, Materie politiche per rapporto all'estero, Consolati nazionali, Barcellona, mazzo 1, 12 settembre 1821. 


\section{Immagini}

Nella lettera di Bottone compare l'atteggiamento anticlericale che caratterizza i due fratelli, ma la relazione complessa con la comunità ospitante, che non si era comunque dimostrata semplice anche nella testimonianza di Garda ci sembra un dato comune. Ricorda ad esempio Giuseppe Avezzana, in quel momento dislocato nei dintorni di Ronda, in Andalusia:

E in verità lo spirito pubblico delle plebi ci era avverso: i preti e i frati, che erano un nugolo in quei paesi, ce lo avevano con arti infami talmente concitato contro, che dovemmo conquistare palmo a palmo il terreno [...] difatti questo parroco venne sorpreso dai miei soldati, mentre dal campanile faceva segnali con un fazzoletto bianco a quei masnadieri: sicchè, afferatelo con indignazione per le gambe lo capovolsero giù sulla strada, dove di subito morì [...]. In quei paesi le risorgenti guerre civili, dove i partiti si contendono il trionfo, hanno così inferocito gli animi, che il sangue non fa più ribrezzo. ${ }^{49}$

Sorisio cerca di spiegare i motivi di frizione tra le due comunità facendo osservazioni antropologiche:

I catalani sono pure figli di quella razza iberica che per la sua fierezza e per il suo amore eccessivo nazionale fecero prodigi di valore contro i barbari loro nemici conservando sempre un odio tenace contro i forestieri. Queste loro qualità quantunque isterilite dai preti li spinsero in ogni tempo a fare ogni sorta di sacrifici per conseguire la libertà e mantenere la loro indipendenza. Tanta è la loro avversione per gli stranieri che persone civili ed istruite, le quali mi professavano una certa amicizia, mi dicevano di non potermi affezionare perché ero straniero. ${ }^{50}$

Fermiamoci allora sull'immagine della nazione e delle persone che le scritture degli esuli ci rimandano riguardo la Spagna e attraverso essa della patria lontana. A Girona si pubblica la Cantata Patriottica di Luigi Monteggia, sfortunata previsione dell'esito del periodo costituzionale. Scritta nel momento delle azioni contro i realisti, nelle due parti del poema si alternano i versi di quattro protagonisti, Libertà, Genio d'Italia, Vecchio contadino spagnolo e il Coro, che disegnano un quadro vincente dell'unione dei popoli che lottano per la libertà. Nel Genio d'Italia c'è la rappresentazione degli esuli approdati:

Ti bacio o suol beato / Fecondo ognor d'Eroi: / Mantenga amico il fato / L'ardir de'figli tuoi.

Se la mia cara Italia / Sarà felice un dì, / Lo debba a questo popolo / Che un tanto esempio offrì.

49. AvezZANa, I miei ricordi, cit., p. 25-26.

50. Memorie Sorisio, p. 24. 
Insieme a Libertà incontra il contadino che rappresenta la saggezza di una società che riconosce i diritti ma che non eccede: «il popol non trascorra / oltre i limiti suoì. L'armonia è però messa in discussione dai ribelli, che uccidono i figli del contadino ma sono sconfitti grazie anche all'intervento armato del Genio d'Italia. A due voci cantano "Europa scuotiti: / perché temer? / Di opporti ai despoti / Ecco il sentiens. ${ }^{51}$ L'auspicio non si realizza ma l'opera può servire ad introdurre altre riflessioni. Partendo dalla considerazione che l'immagine nazionale nasca anche come riflesso delle immagini altrui (per opposizione o per analogia) è stato sottolineato l'interesse di studiare

la ricezione dello stereotipo romantico da parte dei paesi che ne furono oggetto e più concretamente i meccanismi adoperati da essi per introiettare le rispettive immagini man mano che si acutizzava il conflitto fra fedeltà al passato e modernità borghese. Un'operazione valida soprattutto nel caso dell'Italia, cui l'alter ego spagnolo servì in vario modo da specchio e da discrimine per elaborare il proprio autoritratto risorgimentale. ${ }^{52}$

La stessa autrice sottolinea come l'elogio dell'Alfieri dell'eroismo iberico avesse alimentato una immagine assai positiva della Spagna già nell'Italia prerisorgimentale e che poi «i moti liberali conferirono valore esemplare alla resistenza contro Napoleone e alla successiva Costituzione di Cadice», basta ricordare l'invidia e l'ammirazione di Berchet per le qualità iberiche : "puntiglio d'onore», «l'entusiasmo del popolo» e «l'amor patrio». ${ }^{53}$ Quando l'Italia si inoltreà nel processo risorgimentale, la sua immagine per la Spagna diverrà, secondo l'analisi della Muñiz, sempre più sfuggente rendendo più problematico il tentativo del rispecchiamento. E quindi al consenso riscosso negli ambienti letterari dagli esuli del gruppo vicino al «Conciliatore» approdati nella Catalogna durante il triennio liberale, ${ }^{54}$ va accompagnata la considerazione

51. Luigi Monteggia, Cantata patriottica, Gerona: A. Oliva, 1822. L'opera è dedicata a Lopecio "como un testimonio de mi amistad y aprecio». Su questo lavoro di Monteggia vedi Albertocchi, Exiliats italians a Catalunya a començament del segle XIX, cit.

52. MARIA DE LAS Nieves MuÑIz MuÑIZ, «Italia-Spagna: l'immagine riflessa», in Maria Silvia Tatti (acd), Italia e Italie. Fra rivoluzione e Restaurazione. Atti del Convegno di Studi, Roma, Bulzoni, 1999, p.161-180, p. 163.

53. MuNIIZ, p. 165 citazione di Berchet tratta da «Il Conciliatore», edito da VitTORE BRANCA, Firenze: Le Monnier, 1954, vol. III, p. 183.

54. Muñiz cita, a conforto della tesi di una versione debole del romanticismo conciliante e cattolico importata dall'Italia nella Catalogna attraverso le riviste «El Europeo» e il «Vapor», il giudizio di Macrì: "Questo impeto catalano del "Vapor" è davvero preoccupante, gonfiato il Grossi, e quindi il Manzoni, nelle tube dei Puritani (non ancora l'Aida), aggrovigliato tra scenografie neogotiche e neobarocche (con tentata assimilazione ispanica nelle analogie del Greco e dello Spagnoletto)", (ORESTE MACRÌ, Varia fortuna del Manzoni in terre iberiche, Ravenna, Longo, 1976, p. 21). Ricorda però che qualche decennio dopo si verificarono esempi di "garibaldismo letterario» e la sua analisi fa riferimento a Victor Balaguer, soldato nella campagna del 1859 e autore di un Cant a Garibaldi dove mitologia liberale e ideali nazionalisti catalani si mescolavano in modo inestricabile. 
che quel romanticismo conciliante finì per alimentare gli spiriti reazionari delle generazioni successive (quanto ami esplicita la contrapposizione stabilita da Menendez Pelayo tra i modelli imperanti dopo il 1834 - Manzoni, Pellico, Grossi, D’Azeglio- a quelli validi per gli uomini del 1812 e del 1820, «educados a los pechos de la Enciclopedia y de Bentham») e servì infine a rafforzare l'immagine tradizionale di una Spagna cattolica, monarchica e calderoniana per natura. ${ }^{55}$

Questo primato spagnolo dell'esempio armato si unisce a volte ad una tentazione di fagocitare il paese fratello: come nel caso di Moreno Guerra, deputato di sinistra alle Cortes nel Trienio, che argomenta così la richiesta di appoggio militare ai liberali italiani: «Nápoles es una parte de España, como Cataluña, y debemos auxiliarla», "Nápoles es como una cosa propia; todavía está la sangre mezclada entre los de Nápoles y los de Madrid» (2 aprile 1821). Così possiamo capire come, dopo la sconfitta dei moti costituzionali, per gli italiani la Spagna non rappresenti solo uno spazio di rifugio ma anche la concretizzazione di una Nazione Ideale, per rimanere alla definizione di Carr. Oltre la trasfigurazione mitica è un forte vettore romantico come ci viene rimandato dalle lettere ai familiari degli esiliati. "Qui tutto è libero, tutto unito; gli uomini, le donne, le pietre, le bestie ed i monti, tutto, tutto altro non esala che libertà, diva gradita alle anime pure e ben nate». ${ }^{56}$ Angelo Ravizza poi invitava i parenti a partire: "venite in Spagna o diventate Spagnuoli». 57

\section{Il genere della patria}

I Barcellonesi ed in generale i Catalani sono di bella statura, ben fatti, e ne'
lor moti agilissimi. Occhi neri, nera capellatura, color bruno, spirito pronto e
vivace. Le donne se le metti a petto alle Inglesi le diresti brune; ma in con-
fronto con l'altre di Spagna son bianchissime; di maniere molto semplici, di
piacevole ingenuità nel conversare, ed amabilissime. Tanto le maritate, quan-
to le zitelle, veston per lo più gonna di seta o d'un misto di seta e lana color
nero, con semplice ma bella assettatura alla persona, ed alquanto corta, in
modo da lasciar vedere il piede ed un pochetto della gamba, volendo in ciò
imitare le altre Spagnuole, ed in ispecie le donne d'Andalusia, senza però
averne lo stesso motivo; perchè ove quelle il fanno per certa ambizione e
vaghezza di mostrare un piedino ed una gamba che pajon fatte a pennello, le
Catalane invece ti metton fuori un cotal piede, ch'a vederlo spontanea nasce

55. Muñiz, cit., p. 175. citazione tratta da Marcelino Menendez Pelayo, Quadrado y sus obras (1893), in, Discursos, Madrid: Espasa-Calpe, 1942.

56. Lettera dalla Spagna di Giuseppe Chenna, sottotenente di Alessandria, alla zia, 6 dicembre 1821 (AST, Polizia, Copia lettere private intercettate). Il Chenna sarà poi prigioniero in Francia e si imbarcherà per l'Inghilterra nel 1825.

57. La lettera in AST, Polizia, Mat pol, 1822, II, 10. sottotenente al momento dei moti, tenente per ADB, è a Reus nel 1822. 
l'idea, che vi possan dormir sopra placidi i sonni senza pericolo di cadere. Usano venir in pubblico con nulla in capo, e col solo ornamento d'una ricca e nera capellatura inanellata. Il vestir degli uomini, quel de' signori è quale si vede per tutta Europa, che non offre ormai che una stucchevole uniformità; ma quel del popolo è singolarissimo, ed a certa foggia, che dir si potrebbe celtico-romana. Hanno un gran manto di lana, listato a più colori, cui essi danno il nome di manta, e questo portano sopra una spalla e serve loro non solo da mantello di giorno, ma da coperta di notte, se per qualche accidente son condotti a dormire all' aria aperta; ed a questo fine dall'un de' lati ha i lembi cuciti a guisa di sacco, ove, giacendo, introducono i piedi, e si tiran indosso il rimanente. Calzoni larghi e lunghi, che salgono sino a sommo il petto, di varie stoffe secondo la facoltà di ciascuno, i più ricchi di velluto, $\mathrm{i}$ più poveri di cotone, per lo più color verdognolo, e di state di tela bianca. Giubbetto di velluto o cotone, strettissimo al corpo, e che scende appena mezzo palmo sotto le ascelle, ricamato tutt' intorno o semplicemente orlato di cordoncino di seta o di lana. Farsettino al giubbetto corrispondente, per lo più di seta a vaghi colori. Cingono poi intorno al corpo e sul sommo dell' anche gran sciarpa di lana, ed i più ricchi di seta color rosso, allacciata da un canto, i lembi pendenti sino al ginocchio. Al piede calzari di corda, che non han che la suola, terminati in brevi rivolte, una da vestire un pochetto il tallone, 1'altra, la punta de' piedi, dalle quali muovon nastri, che sorgendo a vario intreccio su per la gamba, sono e sostegno del calzare ed ornamento ad un tempo; molti senza calze di state; e tutti in capo una berretta rossa di lana, stretta alla fronte dinanzi, e scendente di dietro in forma di cono sino a mezzo la schiena. I a qual foggia di vestire tutt' insieme accresce mirabilmente alla vista l'apparente agilità de' loro corpi. Il vestir delle donne volgari non è molto dissimile da quello delle nostre contadine d'Italia; busto e gonna di cotone o seta, e sempre di color diverso, in modo che 1'uno dia risalto all'altro; grembiale di mossolina; nulla in capo od un fazzoletto; capelli raccolti in trecce, cadenti sugli omeri, ovvero avvolte a più giri sul mezzo del capo; gli stessi calzari degli uomini, ed esse pure sovente scalze; aria ed andatura assai disinvolta; e non si veggon mai per via, senza ch' abbian un cestellino sotto al braccio, il quale serve loro, come dicono, di compagnia, e senz' esso non saprebbero ove collocar le braccia e le mani. Van dritte dritte, dimenandosi un cotal poco, e con certa baldanza che va lì lì sui confini dell' impudenza. Fierissime se provocate ; vomitan ingiurie, articolandole con tanta prestezza, che appena vi si può tener dietro colla mente. ${ }^{58}$

Descrizione del piede, e più in generale delle donne, anche nella prosa di Collegno, quando ricorda di essere stato servito dalla figlia di un un oste ad Ocaña, «una giovane, grande più che non lo sogliono essere le spagnuole, fatta a pennello; ed ha occhi degni delle Andaluse». ${ }^{59}$ Il 13 aprile Collegno annota:

58. BeOLCHI, Reminiscenze, cit., p. 53.

59. Giacinto Provana di Collegno, Diario di un viaggio in Spagna nel 1823, alla data del 15 febbraio. Il Diario viene pubblicato su Il Cronista in 16 puntate, praticamente con continuità — se si eccettua il numero 4- dal numero 3 (20 luglio 1856) al numero 19 (10 novembre 1856). Il settimanale torinese venne fondato da Giuseppe Torelli, deputato e amico di Cavour. Al settimanale collaborò, oltre il Torelli che usava lo pseudonimo 
Oggi domenica tutto il sesso gentile della Corogna gira per le chiese ed i passeggi, vestendo quanto ha più di elegante. La bellezza delle Galliziane è meno delicata forse che non quella delle donne di Siviglia o di Madrid; ma anche qui l'occhio vivace, il piedicino microscopico, il portamento orientale ricordano il tipo generale spagnuolo. D. Pedro (quel nostro compagno della Nuestra Señora del Carmen) mi parlava ieri, mentre si aspettava nel porto che ci si venisse a dare pratica, della cortesia delle Corognesi verso i forestieri; i Francesi ed Italiani giunti in fine di marzo ce lo confermano; essi paiono tanti Telemachi presso queste ninfe peninsulari, e non so quale Minerva potrà venirli a rapire per portarli altrove. ${ }^{60}$

Si legano alle ultime parole, con i riferimenti al mondo classico, anche i ricordi di Sorisio, confinato con altri esuli ad Alhucemas, «quella piccola isola era un nudo scoglio elevato a baluardo, su cui si fabbricarono le sole case per gli impiegati governativi e vi sono delle mura guarnite di tre cannoni che qualche volta si sparano contro i marocchini che dalla spiaggia ci bersagliavano». Nell'isola si innamora della figlia del comandante,

nuovo Tancredi nella Gerusalemme liberata di Torquato Tasso andavo in traccia di quella felicità impossibile da raggiungere. Nel pelago di quelle mie agitazioni ed immerso nel dolore di sapere che l'Italia già maestra di civilizzazione stava allora neghittosa e gemente sotto il vergognoso giogo domestico e straniero mi tormentavo talmente che divenni taciturno, solitario, sinchè ammalai costretto di ricoverarmi all'ospedale ove fui riconosciuto dal Medico affetto di scorbuto creduto da alcuni attaccaticcio per cui l'infermiere mi serviva con diffidenza. ${ }^{61}$

Cerchiamo di generalizzare l'argomento. Giovanni Allegra aveva il volto scuro ma, secondo il biografo, tutt'altro che brutto, e quindi esercitava fascino sulle donne catalane «i cui sguardi vengono incontro fissi e diritti come punte di spade». ${ }^{62}$ Il rapporto con il genere femminile che qui ho citato non è un elemento di grande presenza nella memorialistica degli esuli ma dove appare introduce certamente un discorso che ha assunto un peso notevole anche nella recentissima produzione storiografica. ${ }^{63} \mathrm{Ci}$ riferiamo alla relazione tra patria e generi sessuali, tra nazione e sentimenti nel loro farsi concreti. Secondo Banti

Ciro D’Arco, anche Massimo D’Azeglio. Durò solo due anni, anche avendo un discreto pubblico. La citazione è nel n. 11, p. 18 .

60. Collegno, Diario di un viaggio in Spagna nel 1823, cit., n. 16, p. 46.

61. Sorisio, Memorie, cit., p. 56-57.

62. Colli, L'avvocato Giovanni Allegra, cit., p. 57.

63. Per tutti vedi Alberto Mario Banti, La nazione del Risorgimento. Parentela, santità e onore alle origini dell'Italia unita, Torino, Einaudi, 2000 e il volume degli Annali della Storia d'Italia Einuadi, Il Risorgimento, Torino, Einaudi, 2007, curati da Banti e Paul Ginsborg, in particolare i saggi di GINSBORG, Romanticismo e Risorgimento: l'io, l'amore e la nazione; di Marta Bonsanti, Amore familiare, amore romantico e amor di patria, e - nella Parte seconda. Donne e nomini del Risorgimento: esperienze e identità di genere- il saggio di LUCY RIALL, Eroi maschili, virilità e forme della guerra. 
l'identificazione tra patria e donna, eredità del triennio giacobino, passa in Italia attraverso l'esercito francese e l'assimilazione di valori e vocabolario: il termine nazione che descrive la comunità fondamentale che legittima l'istituzione si collega così alla parola patria attraverso il ruolo del cittadino e patriottismo si declina così come amore della patria. Questo determina una attribuzione dei ruoli di genere in cui l'ideale militare, e l'impegno politico, hanno la funzione anche di ridefinire il discorso patriottico: «l'allegoria originaria immagina la patria come una donna e una madre». ${ }^{64}$ Agli sguardi femminili catalani, Allegra non era insensibile, ma è troppo preso dall'amore patrio per pensare a quello più prosaico; l'amore per l'Italia gli assorbe tutte «le potenze dell'anima, che poco tempo ho io avuto da dedicare ad altri amori, sicchè le donne tengono parte ben piccola nella storia della mia vita». ${ }^{65} \mathrm{Ma}$ c'è anche un'altra declinazione di questo discorso che Pecchio attribuisce al racconto di una giovane madrilena che gli descriveva Riego nel settembre 1821, cioè il periodo della rimozione dal comando aragonese: «Ora si dice che prenda moglie! Questo mi dispiace. Se ciò fosse, non sarebbe più vero ch'egli non vive che per la patria; ch'egli non ama che la libertà. No, non deve ammogliarsi. Il suo matrimonio mi sembrerebbe una infedeltà alla nazione. Non è egli il suo amante?». ${ }^{66}$

64. BANTI, La nazione del Risorgimento, cit., p. 67.

65. Colli, L'avvocato Giovanni Allegra, cit., p. 57. Secondo Colli però era indubbiamente irresistibile «quelle poche volte che il suo severo Ideale gli permise di rivolgere gli occhi al Reale. Ed anche in Ispagna il sorriso di più di una Pàquita, l'occhio fiammeggiante di più di una Consuelo, rischiarò d'un raggio limpido e sereno la scura giornata del povero esule». Impara, attraverso le donne, la lingua.

66. PeCCHIO, Sei mesi in Ispagna, cit., p. 46. 